\title{
Deep-Blue Thermally Activated Delayed Fluorescence
}

\section{(TADF) Emitters for Light-Emitting Electrochemical Cells}

\section{(LEECs)}

Michael.Y. Wong, ${ }^{a}$ Maria-Grazia La-Placa, ${ }^{b}$ Antonio Pertegas, ${ }^{b}$ Henk J. Bolink ${ }^{b}$ and Eli Zysman-Colman *a

${ }^{a}$ Organic Semiconductor Centre, EaStCHEM School of Chemistry, University of St Andrews, St Andrews, Fife, UK, KY16 9ST, Fax: +44-1334 463808; Tel: +44-1334 463826; E-mail: eli.zysman-colman@st-andrews.ac.uk; URL: http://www.zysman-

\section{colman.com}

${ }^{b}$ Instituto de Ciencia Molecular (ICMol), Universidad de Valencia, Catedrático José Beltrán, 2, 46980 Paterna, Spain. E-mail: Henk.bolink@uv.es.

\section{SUPPORTING INFORMATION}

\section{Table of Contents:}

Experimental Section 


\section{Experimental Section}

General Synthetic Procedures. All the commercially available chemicals and reagent grade solvents were used as received. Air-sensitive reactions were performed using standard Schlenk techniques under a nitrogen atmosphere. Anhydrous THF was obtained from a solvent purification system. Flash column chromatography was carried out using silica gel (60 $\AA, 40-63 \mu \mathrm{m})$. Analytical thin-layer-chromatography (TLC) was performed with silica plates with aluminum backings $(250 \mu \mathrm{m}$ with F-254 indicator). TLC visualization was accomplished by $254 / 365 \mathrm{~nm}$ UV lamp. ${ }^{1} \mathrm{H},{ }^{13} \mathrm{C}$ and ${ }^{19} \mathrm{~F}$ NMR spectra in $\mathrm{CDCl}_{3}, \mathrm{CD}_{2} \mathrm{Cl}_{2}$ or DMSO- $d 6$ were recorded on a NMR spectrometer (300 or $400 \mathrm{MHz}$ for ${ }^{1} \mathrm{H}, 76$ or $101 \mathrm{MHz}$ for ${ }^{13} \mathrm{C}$ and $282 \mathrm{MHz}$ for ${ }^{19} \mathrm{~F}$ ). Melting points were measured using open-ended capillaries and are uncorrected. High-resolution mass spectrometry (HRMS) was performed by the EPSRC National Mass Spectrometry Service Centre (NMSSC), Swansea University. Elemental analyses were performed by Mr. Stephen Boyer, London Metropolitan University.

\section{$\mathrm{CzC}_{4} \mathbf{I m}, \mathbf{1}$ :}

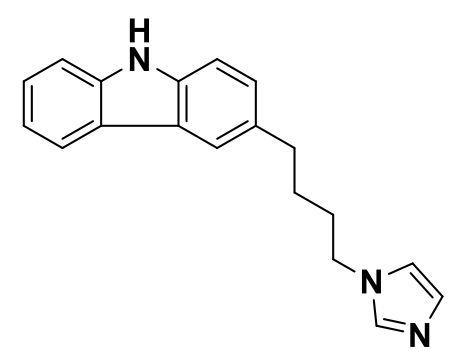

The synthesis of the title compound has been reported previously. ${ }^{1}$

\section{$\left(\mathrm{CzC}_{4} \mathrm{Im}\right)_{2} \mathrm{DPS}, 2$ :}




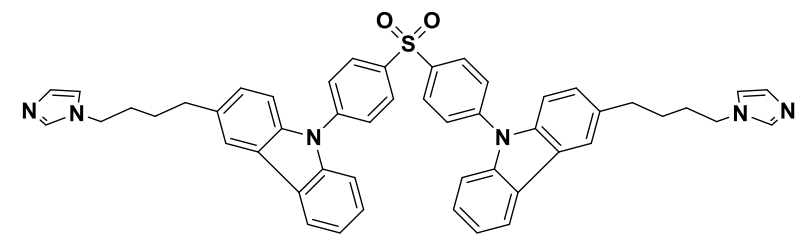

$\mathbf{C z C}_{4} \operatorname{Im}(500 \mathrm{mg}, 1.73 \mathrm{mmol}, 2$ equiv.) and sodium hydride (60\% in mineral oil, 104 mg, 2.60 mmol, 3 equiv.) were mixed in dry DMF (5 mL) and stirred for 15 mins. Bis(4-fluorophenyl)sulfone (220 mg, $0.87 \mathrm{mmol}, 1$ equiv) was added and the mixture was heated at $100^{\circ} \mathrm{C}$ under nitrogen for 6 hours. The reaction mixture was poured into ice water $(5 \mathrm{~mL})$ and extracted with DCM $(3 \times 10 \mathrm{~mL})$. The combined organic layers were dried with anhydrous sodium sulphate and purified by column chromatography (silica, DCM: Hexanes: Triethylamine $=4: 1: 10 \%$ ) to afford the title compound. White solid. Yield: $64 \%$. $\mathbf{R}_{f}: 0.23$ (Acetone : Triethylamine $=9: 1$ on silica). Mp: 115-116 ${ }^{\circ} \mathrm{C} .{ }^{1} \mathbf{H}$ NMR (400 MHz, $\left.\mathbf{C D C l}_{3}\right) \boldsymbol{\delta}$ (ppm): 8.29 (d, J=8.7 Hz, $\left.4 \mathrm{H}\right), 8.13$ (d, $J=7.6 \mathrm{~Hz}, 2 \mathrm{H}), 7.91(\mathrm{~s}, 2 \mathrm{H}), 7.84(\mathrm{~d}, J=8.7,4 \mathrm{H}), 7.63-7.43(\mathrm{~m}, 8 \mathrm{H}), 7.35(\mathrm{t}, \mathrm{J}=$ $7.9 \mathrm{~Hz}, 2 \mathrm{H}), 7.23(\mathrm{dd}, J=8.4,1.6 \mathrm{~Hz}, 2 \mathrm{H}), 7.08(\mathrm{~s}, 2 \mathrm{H}), 6.92(\mathrm{~s}, 2 \mathrm{H}), 3.98$ (t, d = $6.9 \mathrm{~Hz}, 2 \mathrm{H}), 2.85(\mathrm{t}, \mathrm{J}=7.3 \mathrm{~Hz}, 2 \mathrm{H}), 1.92-1.85(\mathrm{~m}, 2 \mathrm{H}), 1.78-1.71(\mathrm{~m}, 2 \mathrm{H}) .{ }^{13} \mathrm{C}$ NMR (101 MHz, $\mathbf{C D C l}_{\mathbf{3}}$ ) $\boldsymbol{\delta}$ (ppm): 142.8, 140.2, 139.2, 138.5, 134.4, 129.7, 127.0, $126.9,126.4,124.2,123.9,121.0,120.5,119.9,109.7,109.6,47.0,35.3,30.6,29.0$. HR-MS (ESI) [M+H] ${ }^{+}$Calculated: $\left(\mathrm{C}_{50} \mathrm{H}_{44} \mathrm{~N}_{6} \mathrm{O}_{2} \mathrm{~S}\right)$ 793.3319; Found: 793.3322 .

\section{imCzDPS:}

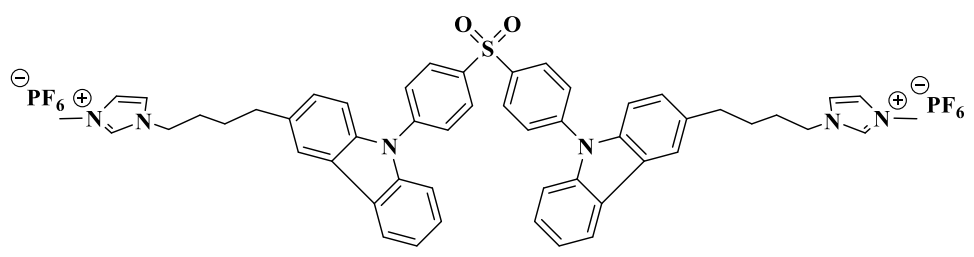

$\left(\mathbf{C}_{\mathbf{Z}} \mathbf{C}_{4} \mathbf{I m}\right) \mathbf{D P S}$ (200 mg, $0.25 \mathrm{mmol}, 1$ equiv.) and methyl iodide (108 mg, $0.75 \mathrm{mmol}$, 3 equiv.) was mixed in acetonitrile $(5 \mathrm{~mL})$ and heated at $40{ }^{\circ} \mathrm{C}$ for 2 hours. After 
cooling, the reaction mixture was poured into saturated aqueous solution $(10 \mathrm{~mL})$ of ammonium hexafluorophosphate to obtain the title compound which was then washed with distilled water and dried thoroughly. White solid. Yield: 96\%. Mp: decomposed at $187{ }^{\circ} \mathrm{C} .{ }^{1} \mathbf{H}$ NMR (300 MHz, DMSO-d6) $\boldsymbol{\delta}$ (ppm): 9.08 (s, $\left.2 \mathrm{H}\right), 8.33$ (d, $J=8.6$ Hz, $4 \mathrm{H}), 8.21(\mathrm{~d}, J=7.7 \mathrm{~Hz}, 2 \mathrm{H}), 8.06(\mathrm{~s}, 2 \mathrm{H}), 7.97(\mathrm{~d}, J=8.7,4 \mathrm{H}), 7.76(\mathrm{~s}, 2 \mathrm{H})$, $7.68(\mathrm{~s}, 2 \mathrm{H}), 7.56-7.27(\mathrm{~m}, 10 \mathrm{H}), 4.20(\mathrm{t}, J=7.0,4 \mathrm{H}), 3.82(\mathrm{~s}, 3 \mathrm{H}), 2.80(\mathrm{t}, J=7.2$ Hz, 4 H), 1.89-1.79 (m, 4 H), 1.68-1.59 (m, 4 H). ${ }^{13}$ C NMR (76 MHz, DMSO-d6) $\delta$ (ppm): 146.2, 143.5, 140.9, 139.9, 139.2, 135.0, 130.4, 127.6, 127.5, 127.0, 124.9, 124.5, 122.2, 120.5, 111.0, 47.2, 37.4, 35.5, 30.8, 29.1. ${ }^{19}$ F NMR (282 MHz, DMSOd6) $\delta$ (ppm): -68.9, -71.4. HR-MS (ESI) [M] ${ }^{2+}$ Calculated: $\left(\mathrm{C}_{50} \mathrm{H}_{50} \mathrm{~N}_{6} \mathrm{O}_{2} \mathrm{~S}\right) 411.1852$; Found: 411.1845 .

\section{TPAC $_{4} I m, 3:$}

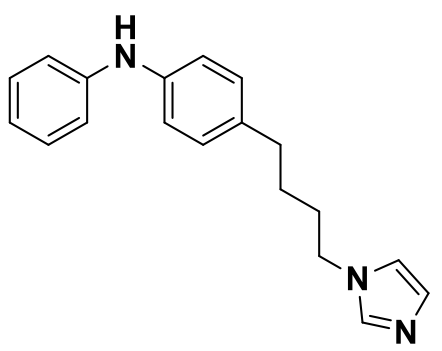

The title compound was accomplished using the same procedure as $\mathbf{C}_{\mathbf{Z}} \mathbf{C}_{\mathbf{4}} \mathbf{I m}, \mathbf{1},{ }^{\mathbf{1}}$ except diphenylamine was used instead of carbazole. White solid. $\mathbf{R}_{f}: 0.16$ (EA on silica). Mp: $134-135{ }^{\circ} \mathrm{C} .{ }^{1} \mathbf{H}$ NMR (300 MHz, $\mathbf{C D C l}_{3}$ ) $\boldsymbol{\delta}$ (ppm): 7.42 (s, $\left.1 \mathrm{H}\right), 7.23$ $7.18(\mathrm{~m}, 2 \mathrm{H}), 7.05(\mathrm{~d}, \mathrm{~J}=8.5 \mathrm{~Hz}, 2 \mathrm{H}), 6.98-6.92(\mathrm{~m}, 7 \mathrm{H}), 3.94(\mathrm{t}, J=7.0,2 \mathrm{H}), 2.59$ (t, $J=6.8,2 \mathrm{H}), 1.86-1.76(\mathrm{~m}, 2 \mathrm{H}), 1.65-1.55$ (m, $2 \mathrm{H}) .{ }^{13} \mathbf{C}$ NMR (76 MHz, CDCl $)$ $\boldsymbol{\delta}$ (ppm): 141.0, 139.2, 138.2, 133.6, 130.5, 127.4, 126.8, 124.5, 124.1, 121.2, 120.6, 120.3 119.8, 111.5, 111.4, 47.4, 30.9, 29.3, 20.1. HR-MS (ESI) [M+H] ${ }^{+}$Calculated: $\left(\mathrm{C}_{19} \mathrm{H}_{22} \mathrm{~N}_{3}\right)$ 292.1808; Found: 292.1806. 
(TPAC 4 Im)DPS, 4:

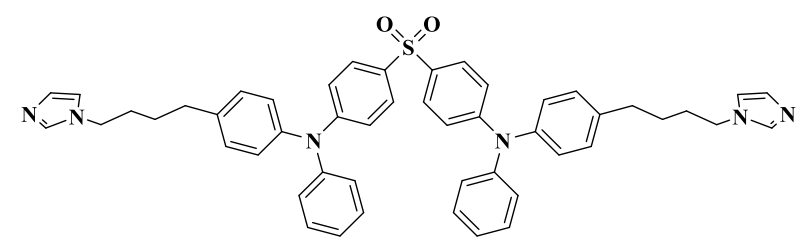

The title compound was synthesized from $\mathbf{T P A C}_{4} \mathbf{I m} \mathbf{3}$ and $\operatorname{Bis}(4-$ fluorophenyl)sulfone in an analogous fashion to $\left(\mathbf{C}_{\mathbf{Z}} \mathbf{C}_{4} \mathbf{I m}\right) \mathbf{D P S}, \mathbf{2}$. White solid. Yield: 42\%. R $\mathbf{R}_{\mathbf{f}}: 0.17$ (Acetone : Triethylamine = 9: 1 on silica). $\mathbf{M p : ~ 9 7 - 9 8 ~}{ }^{\circ} \mathrm{C} .{ }^{1} \mathbf{H}$ NMR (300 MHz, $\left.\mathbf{C D C l}_{3}\right) \boldsymbol{\delta}$ (ppm): 7.43 (s, $\left.1 \mathrm{H}\right)$, 7.19-6.88 (m, $\left.10 \mathrm{H}\right)$, 6.87-6.75 (m, $\left.4 \mathrm{H}\right)$, $3.95(\mathrm{t}, J=7.1 \mathrm{~Hz}, 2 \mathrm{H}), 2.58(\mathrm{t}, J=6.9 \mathrm{~Hz}, 2 \mathrm{H}), 1.85-1.76(\mathrm{~m}, 2 \mathrm{H}), 1.64-1.53(\mathrm{~m}, 2$ H). ${ }^{13}$ C NMR (76 MHz, $\mathbf{C D C l}_{3}$ ) $\boldsymbol{\delta}$ (ppm): 141.1, 140.2, 139.5, 138.4, 135.7, 134.7, $134.0,130.6,127.9,127.8,127.3,125.1,124.7,121.8,121.4,120.8,110.4,47.3,30.8$, 29.2, 19.1.

imDPADPS:

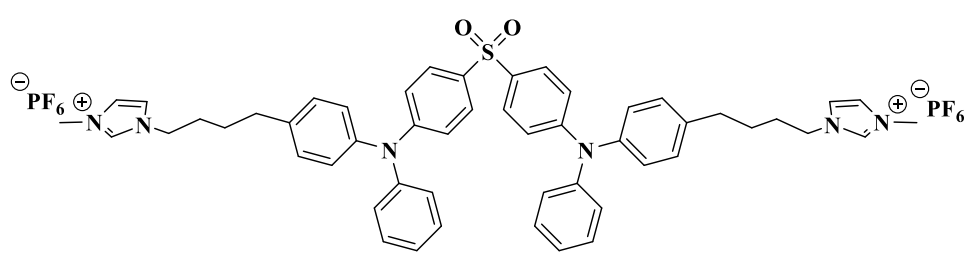

The title compound was synthesized from $\left(\mathbf{T P A C} \mathbf{C}_{4} \mathbf{I m}\right)$ DPS, $\mathbf{4}$ in an analogous fashion to imCzDPS. White solid. Yield: 93\%. Mp: 171-172 ${ }^{\circ} \mathrm{C} .{ }^{\mathbf{1}} \mathbf{H}$ NMR (300 MHz, $\left.\mathbf{C D}_{2} \mathbf{C l}_{2}\right) \boldsymbol{\delta}$ (ppm): $8.54(\mathrm{~s}, 2 \mathrm{H}), 7.63(\mathrm{~d}, \mathrm{~J}=8.9 \mathrm{~Hz}, 4 \mathrm{H}), 7.36-7.28(\mathrm{~m}, 8 \mathrm{H})$, 7.18$7.12(\mathrm{~m}, 10 \mathrm{H}), 7.06(\mathrm{~d}, \mathrm{~J}=8.4 \mathrm{~Hz}, 4 \mathrm{H}), 6.94(\mathrm{~d}, \mathrm{~J}=8.9 \mathrm{~Hz}, 4 \mathrm{H}), 4.21$ (t, J = 7.4 Hz, $4 \mathrm{H}), 3.94(\mathrm{~s}, 6 \mathrm{H}), 2.67$ (t, d = 7.0 Hz, $4 \mathrm{H}), 2.00-1.90(\mathrm{~m}, 4 \mathrm{H}), 1.73-1.65$ (m, $4 \mathrm{H})$. ${ }^{13}$ C NMR (76 MHz, DMSO-d6) $\delta$ (ppm): 144.0, 143.0, 142.3, 141.2, 138.4, 137.4, $136.7,133.3,130.5,130.4,129.8,127.6,127.2,124.3,123.8,123.2,112.6,47.9,37.8$, 


\section{0, 29.4, 19.4. ${ }^{19}$ F NMR (282 MHz, $\left.\mathbf{C D}_{2} \mathbf{C l}_{2}\right) \delta$ (ppm): -71.2, -73.7. HR-MS (ESI)}

[M-PF 6$]^{+}$Calculated: $\left(\mathrm{C}_{52} \mathrm{H}_{54} \mathrm{~F}_{6} \mathrm{~N}_{6} \mathrm{O}_{2} \mathrm{PS}\right)$ 917.3665; Found: 917.3657.

Photophysical measurements. Optically dilute solutions of concentrations in the order of $10^{-5}$ or $10^{-6} \mathrm{M}$ were prepared in HPLC grade acetonitrile for absorption and emission analyses. Absorption spectra were recorded at room temperature on a double beam spectrophotometer. Molar absorptivity values were determined by linear regression analysis from at least four solutions of different concentrations within the range of $10^{-4}$ to $10^{-5} \mathrm{M}$. Aerated solutions were prepared by using aerated solvent with prior air bubbling for $5 \mathrm{~min}$ whereas degassed solutions were prepared via five freezepump-thaw cycles prior to emission analysis using a home-made cuvette extended solvent bulb designed for cryogenic degassing. Steady state emission and timeresolved emission spectra were recorded at $298 \mathrm{~K}$. Samples were excited at $360 \mathrm{~nm}$ for steady state measurements and at $378 \mathrm{~nm}$ for time-resolved measurements. Photoluminescence quantum yields for solutions were determined using the optically dilute method $^{2}$ in which four sample solutions with absorbance at $360 \mathrm{~nm}$ being ca. $0.10,0.080,0.060$ and 0.040 were used. Their emission intensities were compared with those of a reference, quinine sulfate, whose quantum yield $\left(\Phi_{\mathrm{r}}\right)$ in $1 \mathrm{~N} \mathrm{H}_{2} \mathrm{SO}_{4}$ was taken as $54.6 \%{ }^{3}$ The quantum yield of sample, $\Phi_{\mathrm{s}}$, can be determined by the equation $\Phi_{\mathrm{s}}=\Phi_{\mathrm{r}}\left(\mathrm{A}_{\mathrm{r}} / \mathrm{A}_{\mathrm{s}}\right)\left(\left(\mathrm{I}_{\mathrm{s}} / \mathrm{I}_{\mathrm{r}}\right)\left(\mathrm{n}_{\mathrm{s}} / \mathrm{n}_{\mathrm{r}}\right)^{2}\right.$, where A stands for the absorbance at the excitation wavelength $\left(\lambda_{\text {exc }}: 360 \mathrm{~nm}\right), \mathrm{I}$ is the integrated area under the corrected emission curve and $\mathrm{n}$ is the refractive index of the solvent, with the subscripts " $\mathrm{s}$ " and " $r$ " representing sample and reference respectively. An integrating sphere was employed for quantum yield measurements for thin film samples.

Electrochemistry measurements. Cyclic Voltammetry (CV) analysis was performed on a potentiostat. Samples were prepared as $\mathrm{MeCN}$ solutions, which were degassed by 
sparging with MeCN-saturated nitrogen gas for 15 minutes prior to measurements. All measurements were performed in $0.1 \mathrm{M} \mathrm{MeCN}$ solution of tetrabutylammonium hexafluorophosphate $\left(n \mathrm{Bu}_{4} \mathrm{NPF}_{6}\right) . \mathrm{An} \mathrm{Ag} / \mathrm{Ag}^{+}$electrode was used as the reference electrode while a platinum electrode and a platinum wire $\left(\mathrm{Pt} / \mathrm{Pt}^{+}\right)$were used as the working electrode and counter electrode, respectively. The redox potentials are reported relative to a saturated calomel electrode (SCE) with a ferrocenium/ferrocene $\left(\mathrm{Fc}^{+} / \mathrm{Fc}\right)$ redox couple as the internal standard $(0.38 \mathrm{~V}$ vs $\mathrm{SCE}){ }^{4}$ 


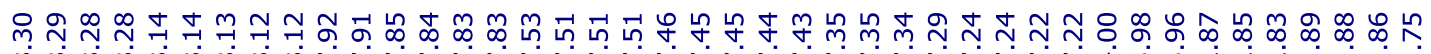

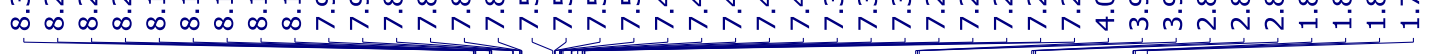

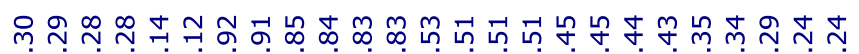

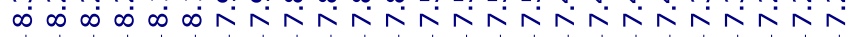
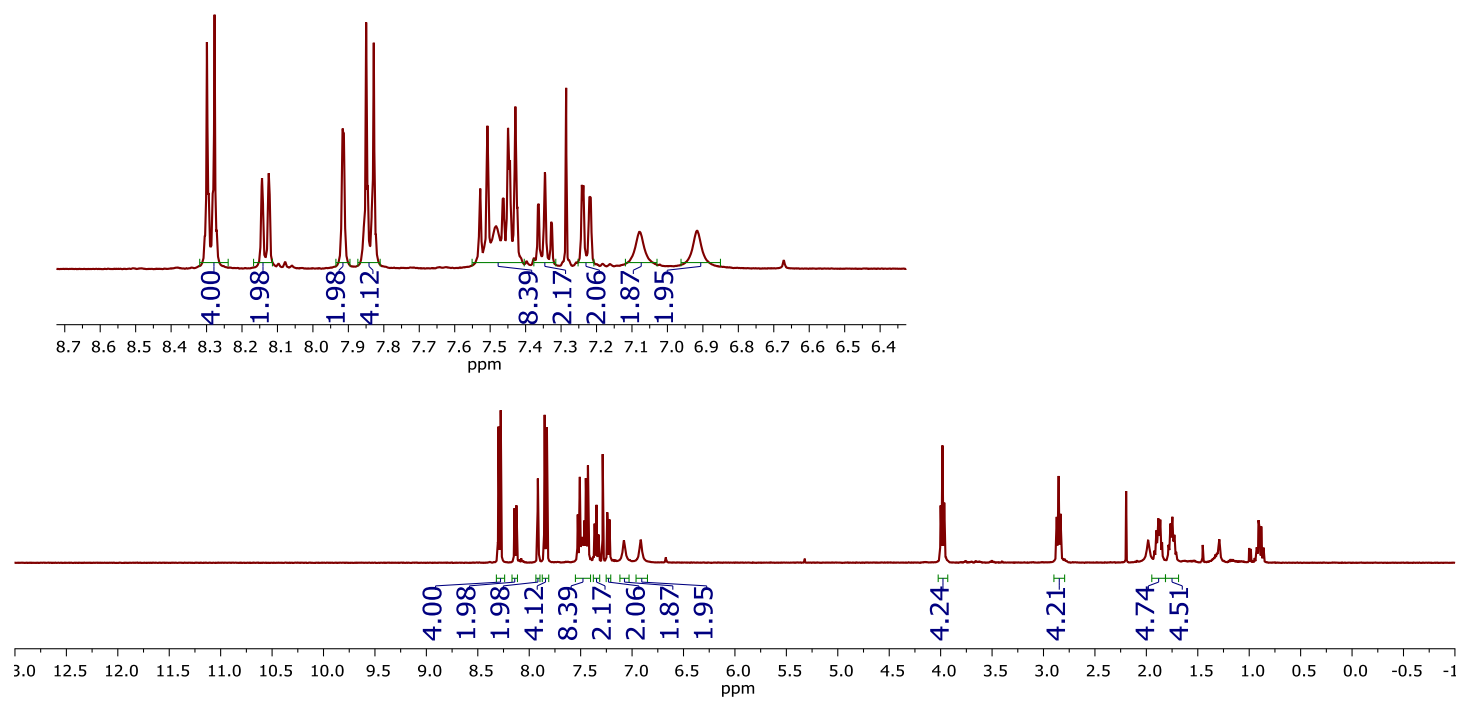

Figure S1. ${ }^{1}$ H NMR spectrum of $\left(\mathbf{C z C}_{4} \mathbf{I m}\right)$ DPS. 


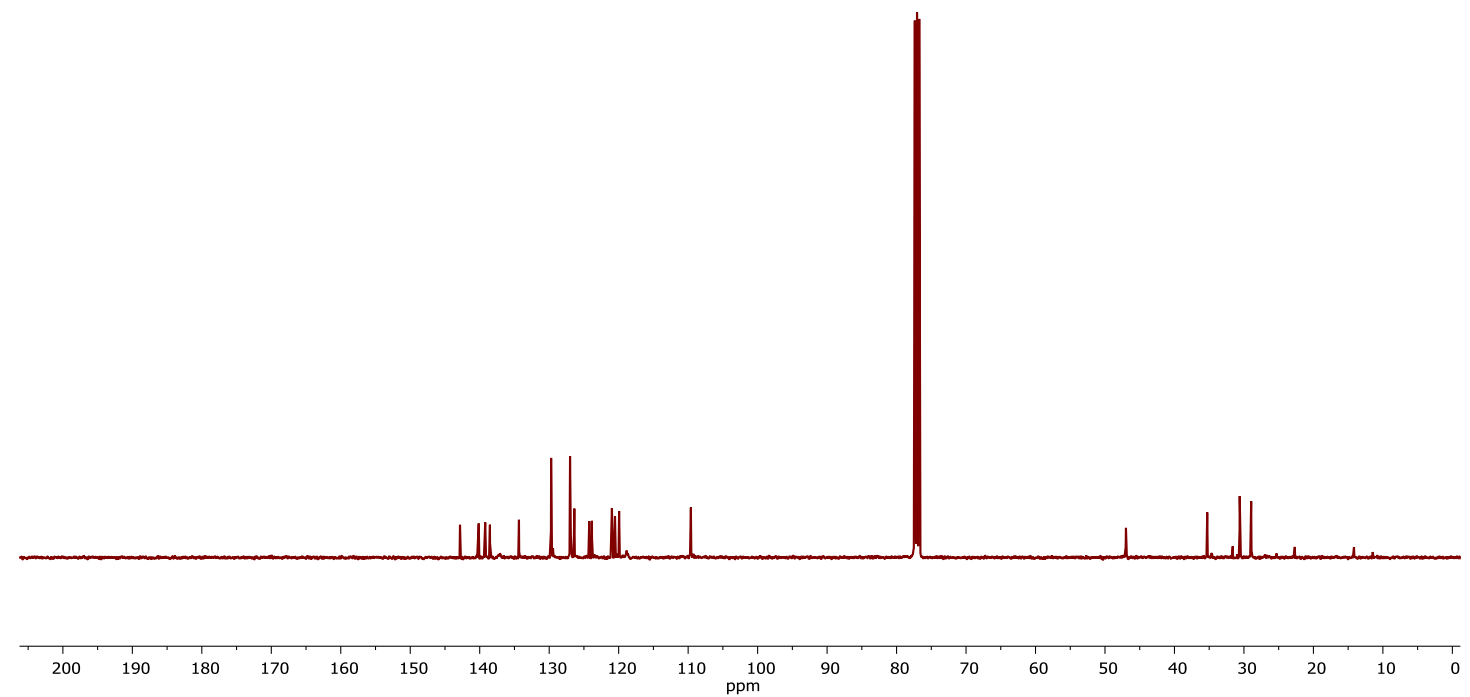

Figure S2. ${ }^{13} \mathrm{C}$ NMR spectrum of $\left(\mathrm{CzC}_{4} \mathrm{Im}\right)$ DPS.

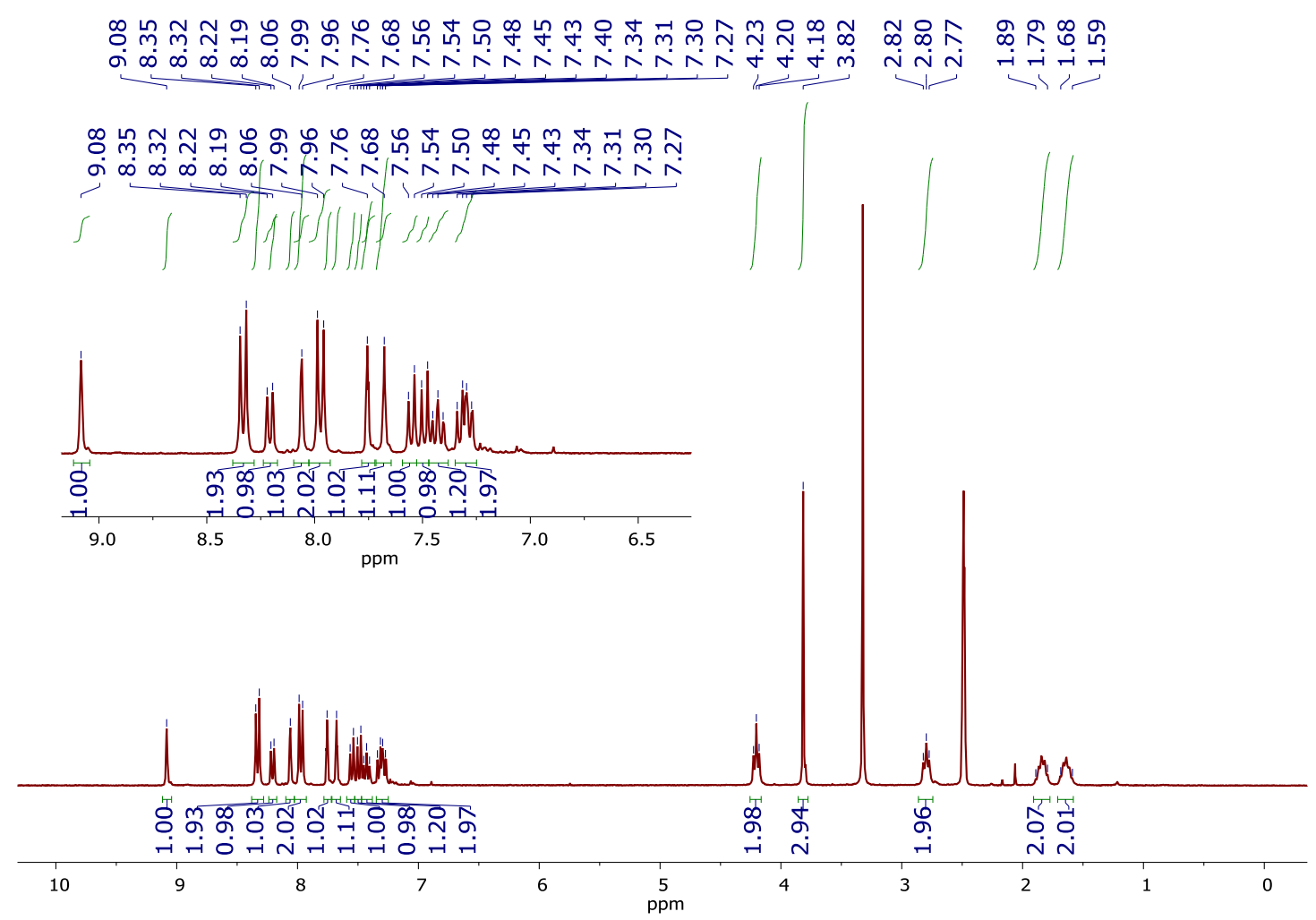

Figure S3. ${ }^{1}$ H NMR spectrum of imCzDPS. 


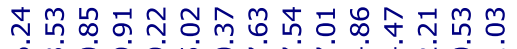

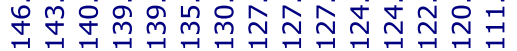

난은

ผึं लें

1 ।

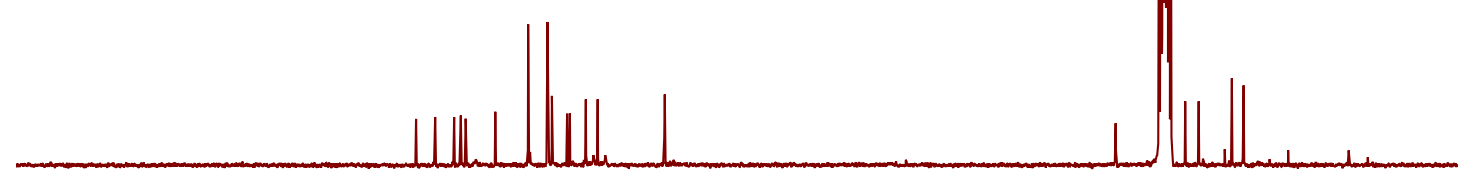

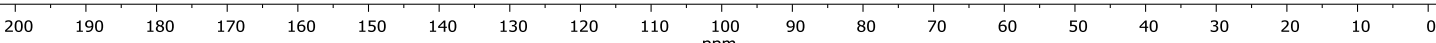

Figure S4. ${ }^{13} \mathrm{C}$ NMR spectrum of imCzDPS.

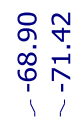

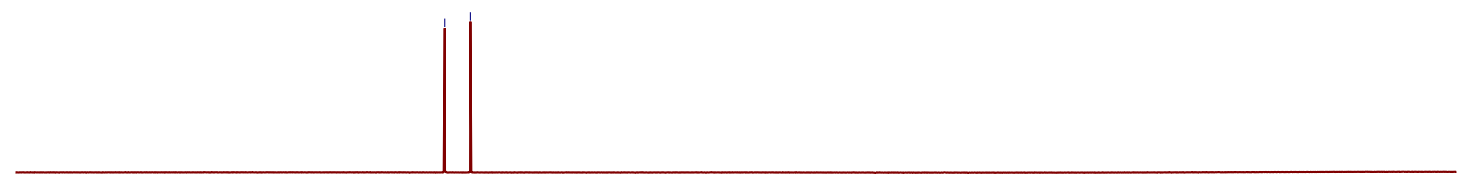

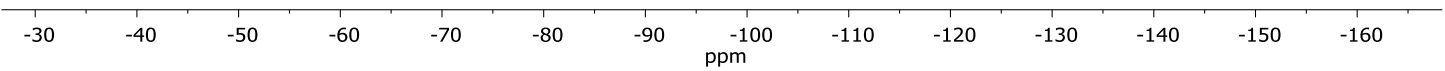

Figure S5. ${ }^{19}$ F NMR spectrum of imCzDPS. 
₹Nํำ

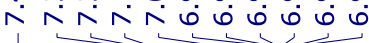
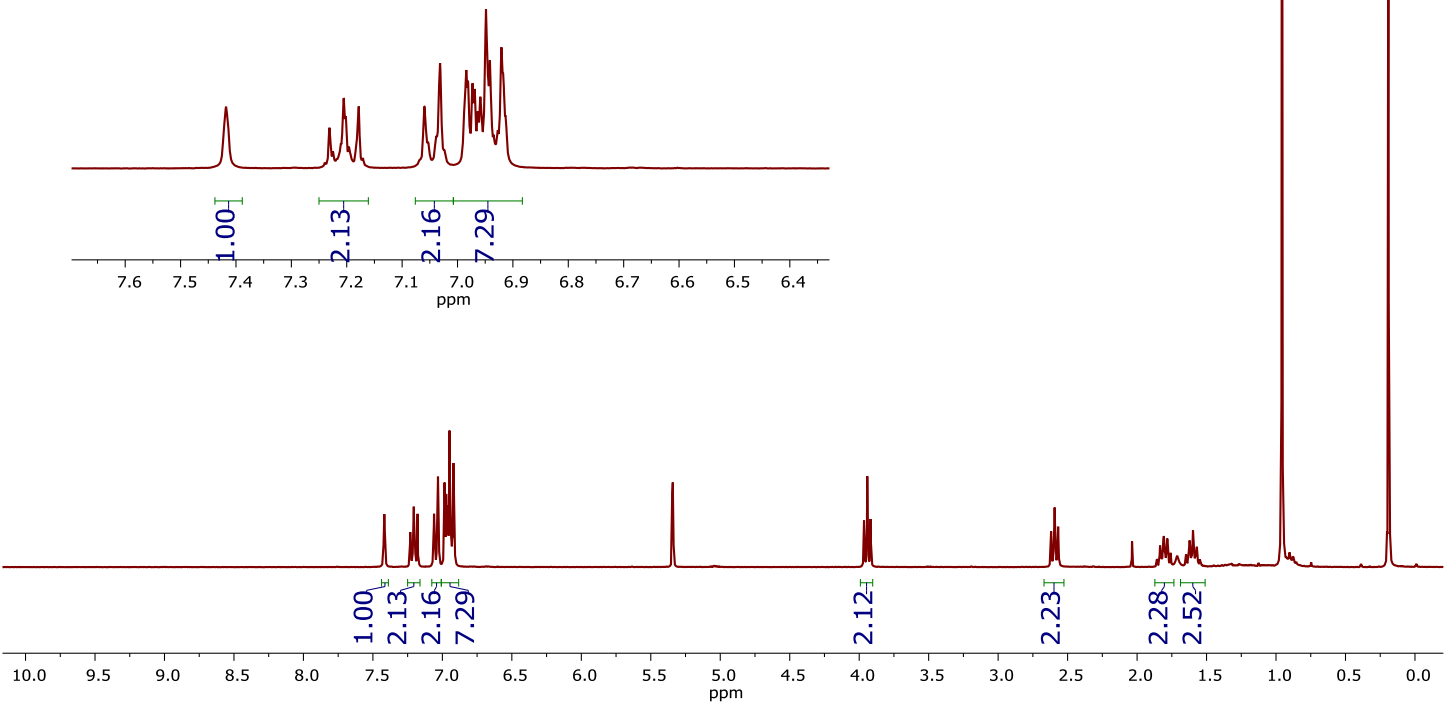

Figure S6. ${ }^{1} \mathrm{H}$ NMR spectrum of $\mathbf{T P A C}_{4} \mathbf{I m}$.

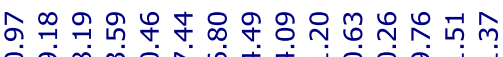

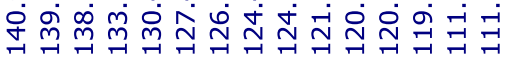

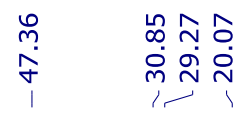
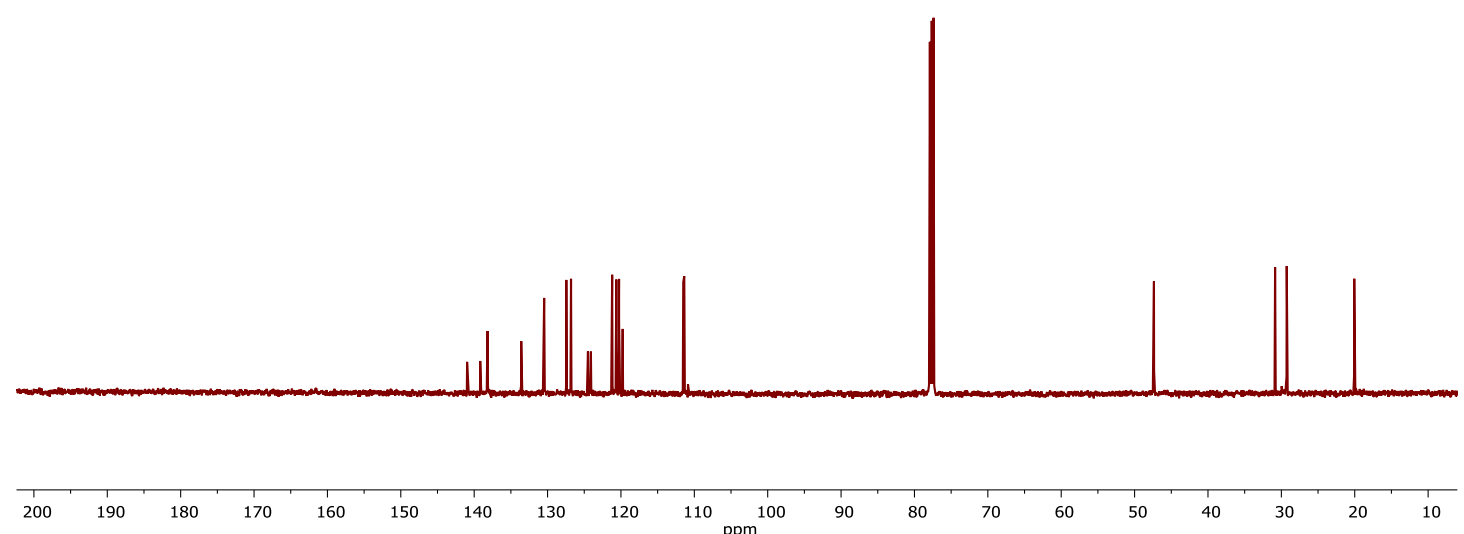

Figure S7. ${ }^{13} \mathrm{C}$ NMR spectrum of $\mathbf{T P A C} \mathbf{C}_{4} \mathbf{I m}$. 


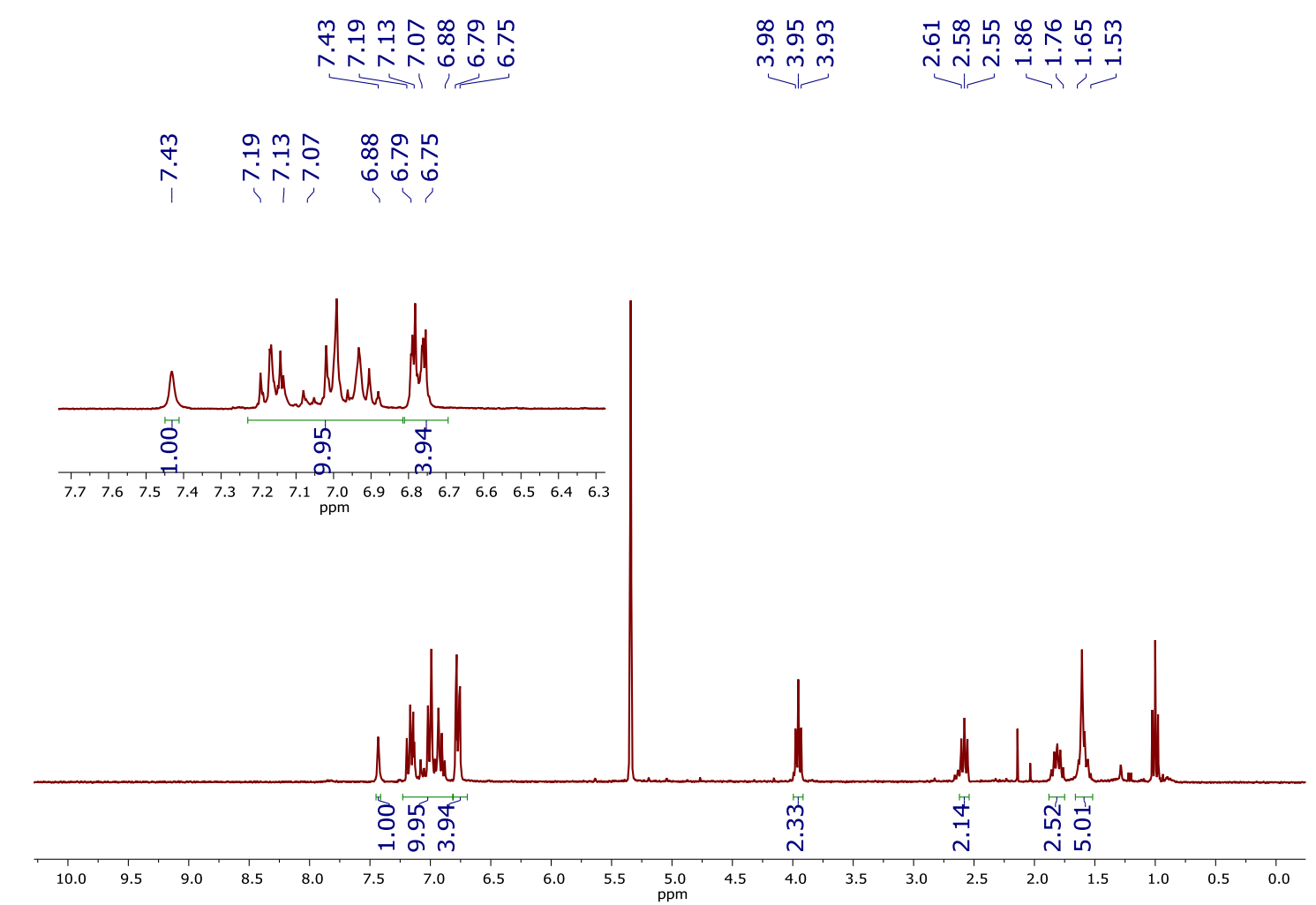

Figure S8. ${ }^{1} \mathrm{H}$ NMR spectrum of $\left(\right.$ TPAC $\left._{4} \mathbf{I m}\right)$ DPS.
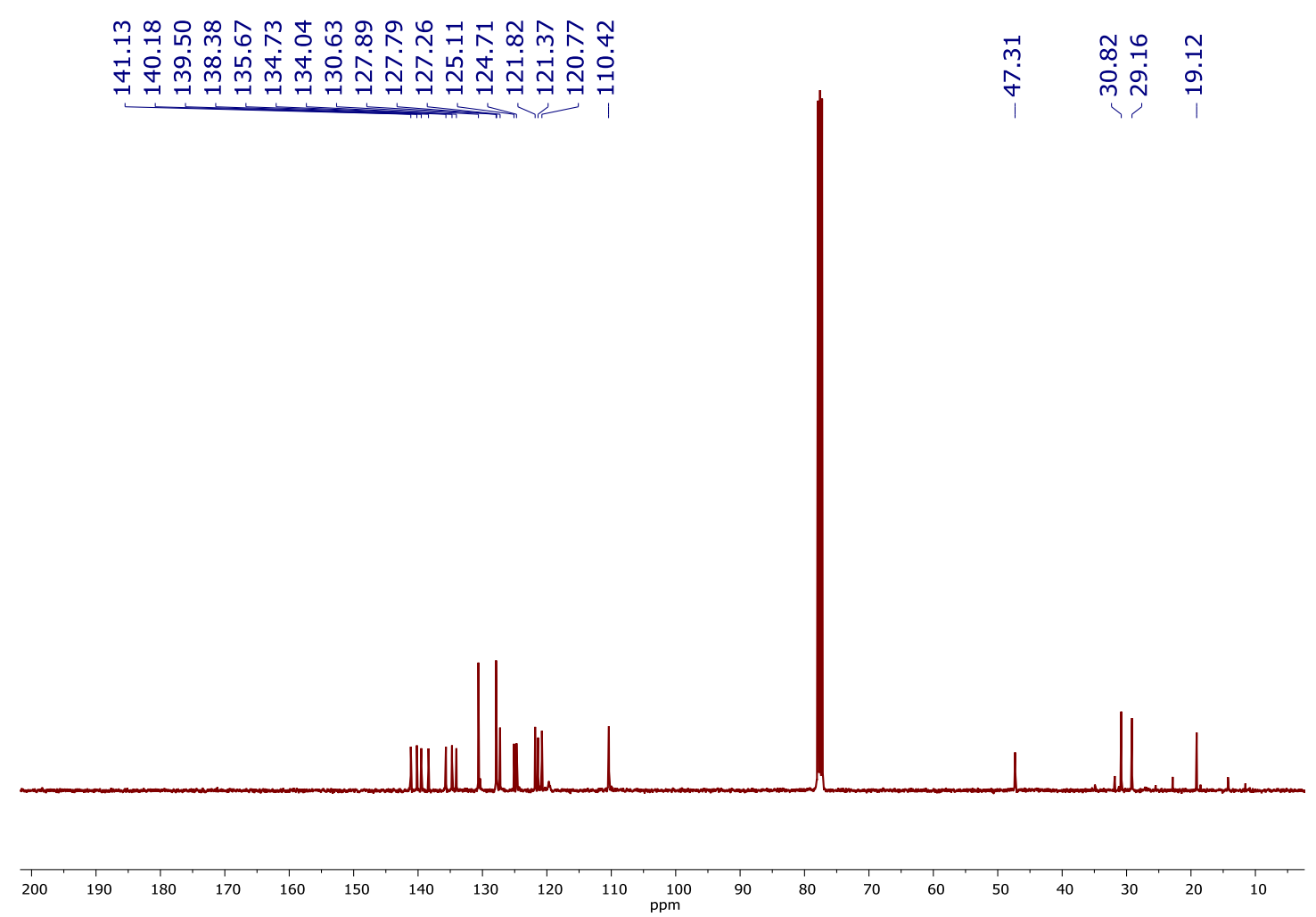

Figure S9. ${ }^{13} \mathrm{C}$ NMR spectrum of (TPAC 4 Im)DPS. 


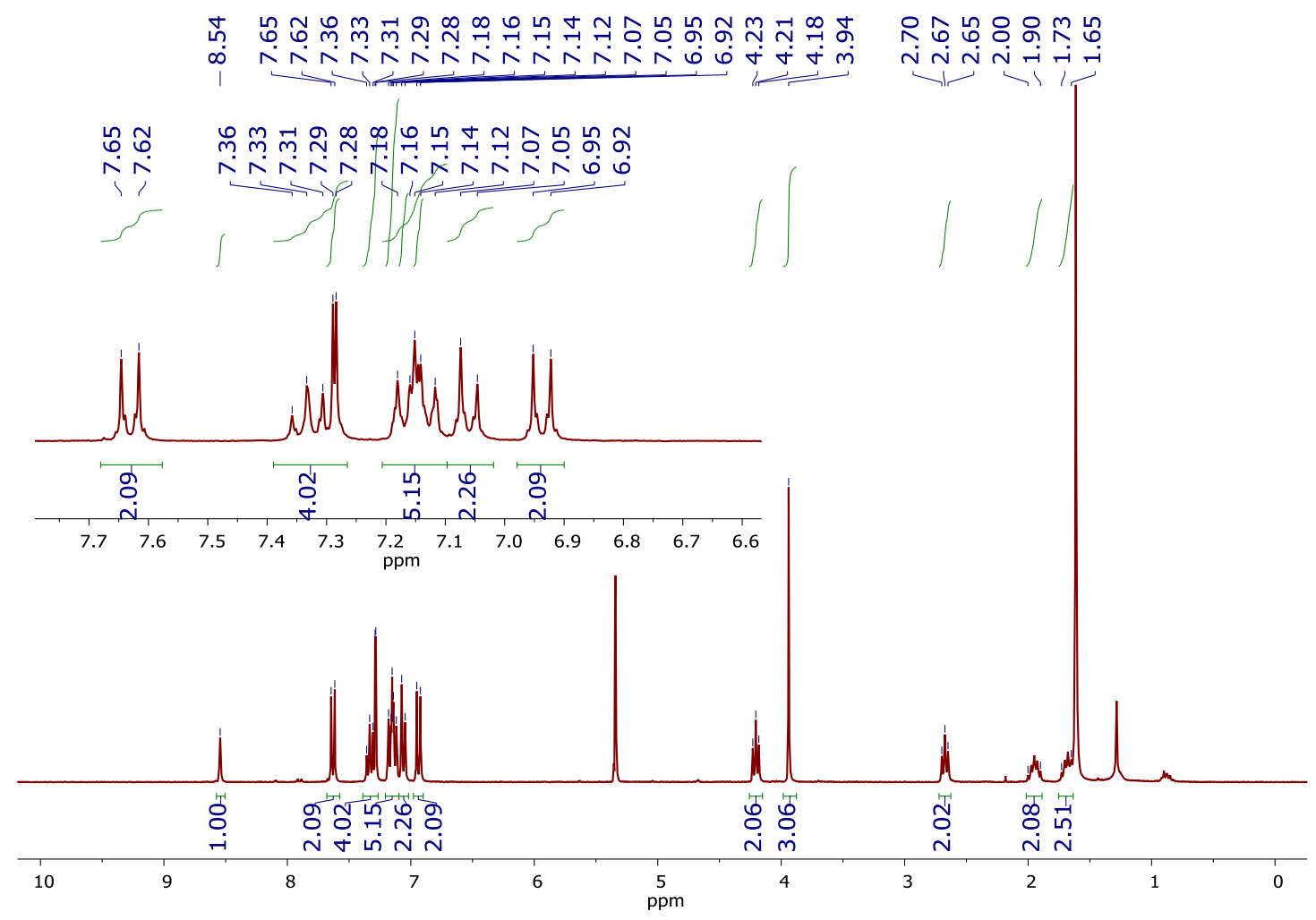

Figure S10. ${ }^{1} \mathrm{H}$ NMR spectrum of imDPADPS.

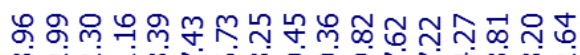

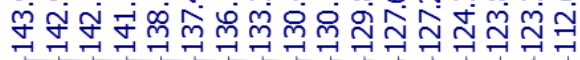
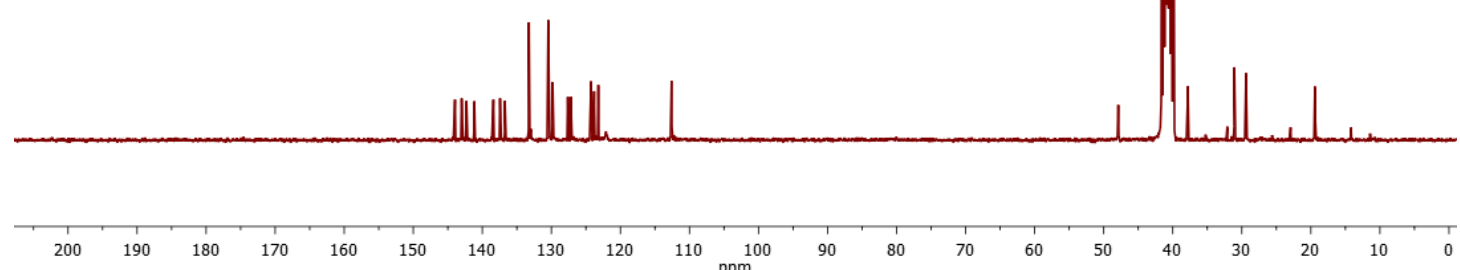

Figure S11. ${ }^{13} \mathrm{C}$ NMR spectrum of imDPADPS. 


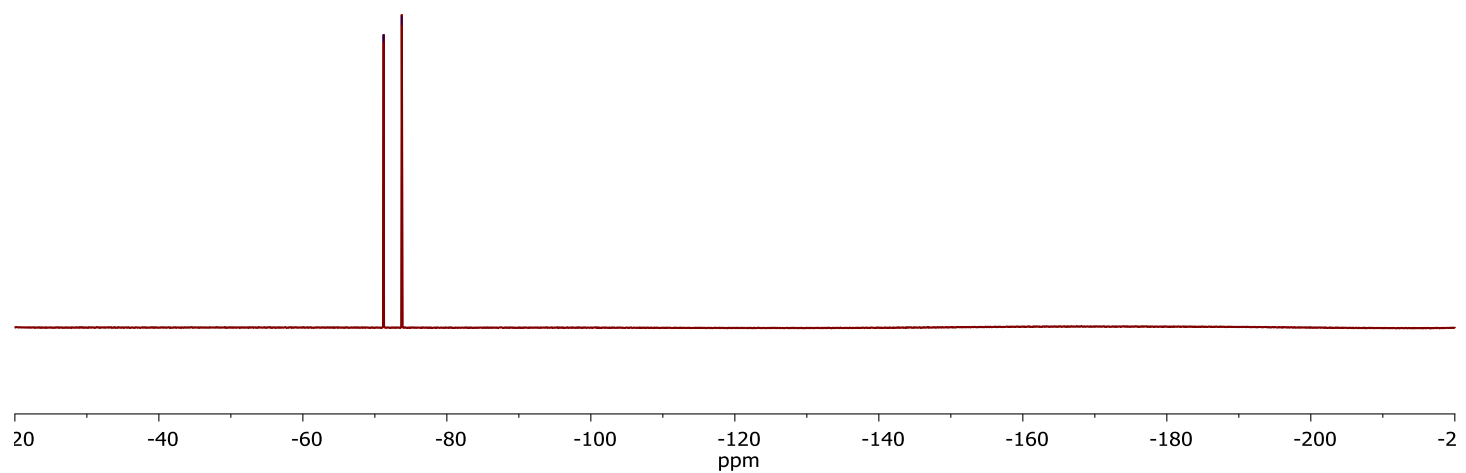

Figure S12. ${ }^{19}$ F NMR spectrum of imDPADPS.

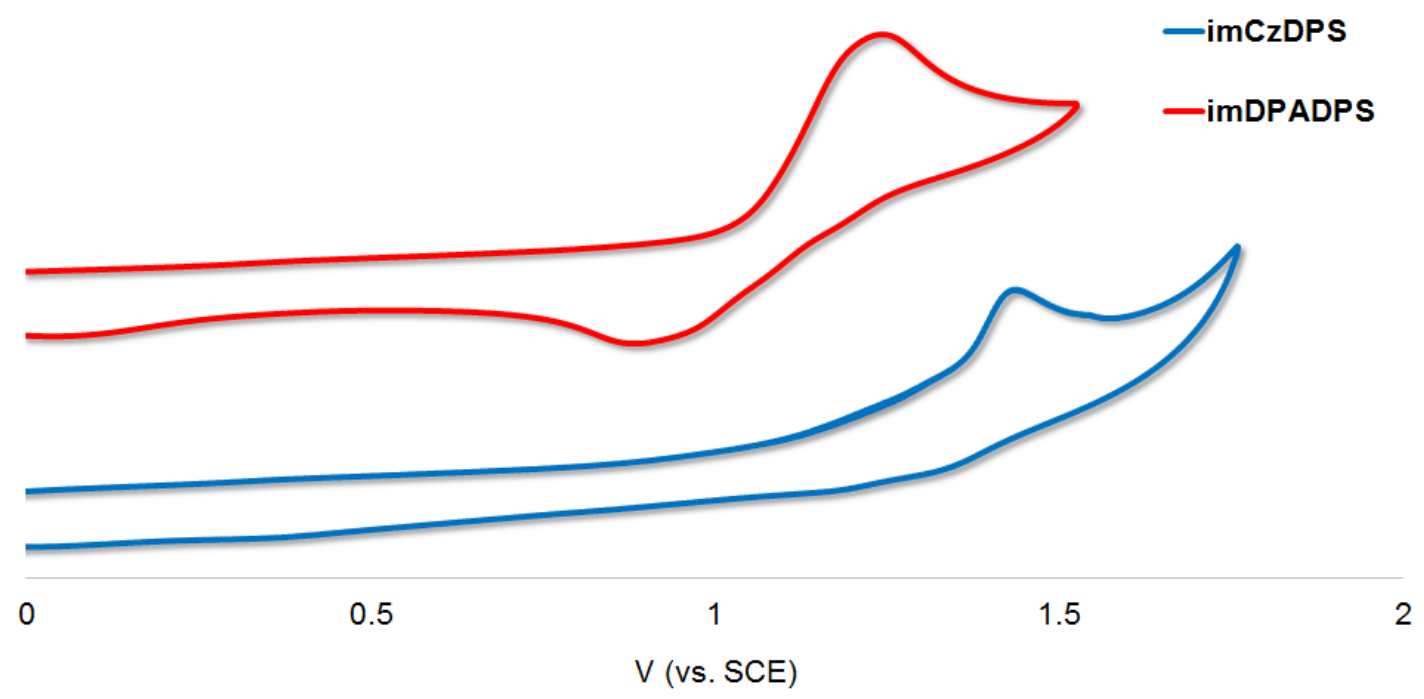

Figure S13. Cyclic voltammograms of imCzDPS and imDPADPS in degassed MeCN under argon with $0.1 \mathrm{M} n-\mathrm{Bu}_{4} \mathrm{NPF}_{6}$ as the supporting electrolyte. 

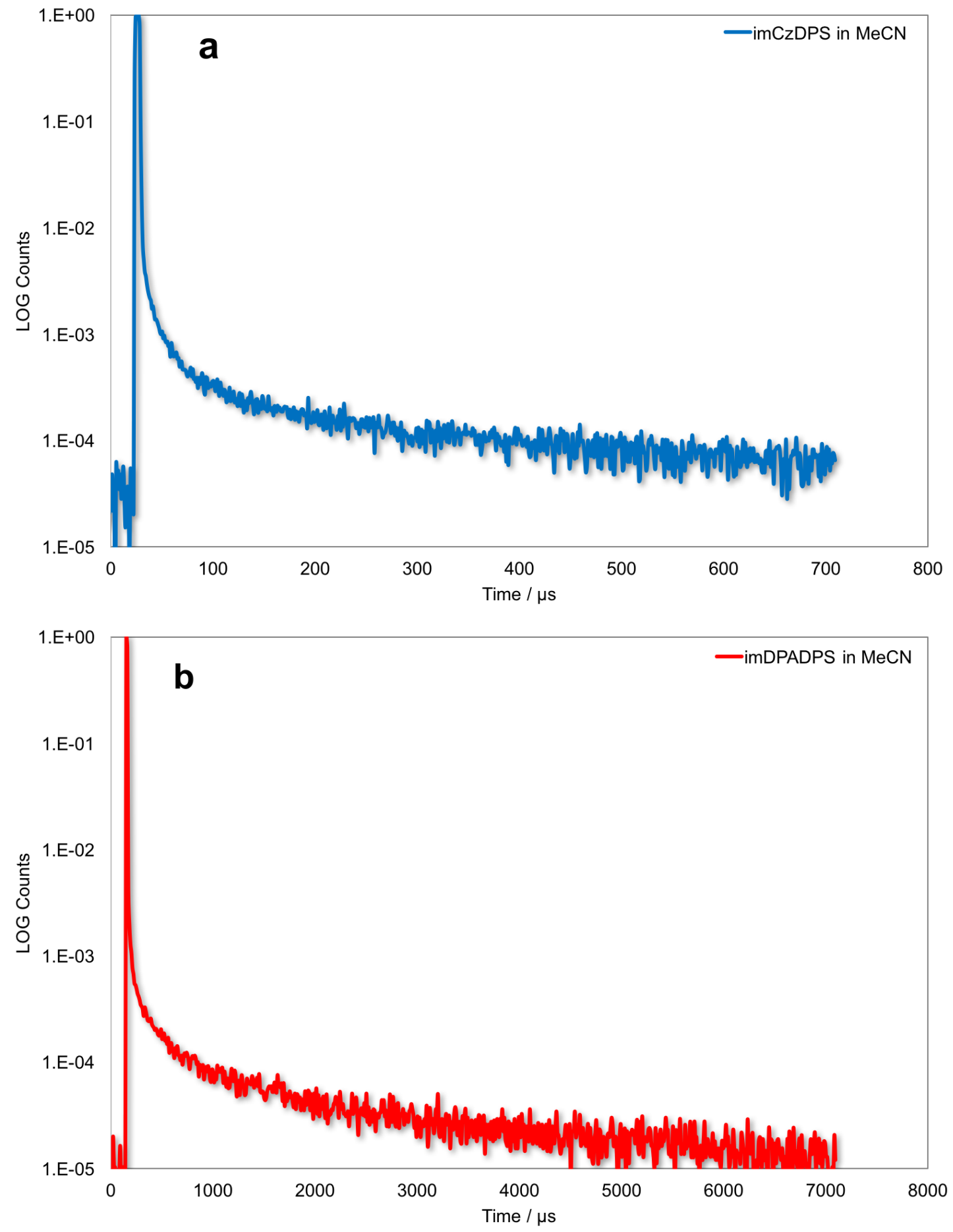

Figure S14. Emission decays of imCzDPS (a) and imDPADPS (b) in degassed $\mathrm{MeCN}$. 


\section{References}

(1). M. Y. Wong, G. J. Hedley, G. Xie, L. S. Kölln, I. D. W. Samuel, A. Pertegás, H. J. Bolink and E. Zysman-Colman, Chem. Mater., 2015, 27, 6535-6542.

(2). G. A. Crosby and J. N. Demas, J. Phys. Chem., 1971, 75, 991-1024.

(3). W. H. Melhuish, J. Phys. Chem., 1961, 65, 229-235.

(4). V. V. Pavlishchuk and A. W. Addison, Inorg. Chim. Acta, 2000, 298, 97-102. 